\title{
MS27-03 | Understanding Two-Dimensional Polymerisation Using Bragg AND DifFUSE X-RAY SCATTERING
}

Weber, Thomas (ETH Zurich, Zürich, CH); Hofer, Gregor (ETH Zurich, Zürich, CH); Simonov, Arkadiy (ETH Zurich, Zürich, CH); Kröger, Martin (ETH Zurich, Zürich, CH); Schlüter, Dieter (ETH Zurich, Zürich, CH)

Two-dimensional polymers are topologically exceptional macromolecules. One approach for synthesising such graphene-analogue polymeric material is the single-crystal-to-single-crystal polymerization method, where the monomers are pre-oriented in a layered 'reactive packing'. In this packing, the polymerization may be triggered by light and it can be reversed by heat treatment or illumination with light of a different wavelength. In this contribution we will present a detailed study of the polymerization and depolymerization mechanisms of a twodimensional polymer based on Bragg and diffuse scattering. The latter was achieved with the 3D-DeltaPDF method using the program Yell.

We could identify the most important factors that made such a complex non-destructive transformation possible. It was found that the incorporated solvent molecules buffer the local strain induced by polymerization through a complex chain of movements. As a result, the accumulated global strain remains small enough to essentially maintain the single crystal state during a complete polymerization/depolymerization cycle. Of particular importance for the understanding of two-dimensional polymers is the development of the connectivity between molecules. The combination of reaction kinetics with real structure information as obtained from diffuse scattering, enabled the development of a polymerisation propagation model. It was found that both, polymerization and depolymerization are self-impeding processes, which lead to a more uniform spatial distribution of the polymer bonds in the intermediate structures than island models. As a result, polymerizationinduced strain does not accumulate locally, but is distributed more evenly over the crystal and allows to preserve the integrity of the single-crystalline state. 